\title{
Numerical solution of nonlinear wave equations in stratified dispersive media
}

\author{
Ch. Karle ${ }^{\text {a }}$, J. Schweitzer ${ }^{b}$, \\ M. Hochbruck ${ }^{\text {b }}$, E.W. Laedke ${ }^{\text {a }}$ K.H. Spatschek ${ }^{\mathrm{a}, *}$ \\ ${ }^{a}$ Institut für Theoretische Physik, Heinrich-Heine-Universität Düsseldorf, \\ D-40225 Düsseldorf, Germany \\ ${ }^{\mathrm{b}}$ Mathematisches Institut, Heinrich-Heine-Universität Düsseldorf, \\ D-40225 Düsseldorf, Germany
}

\begin{abstract}
Nonlinear wave motion in dispersive media is solved numerically. The model applies to laser propagation in a relativistic plasma. The latter causes, besides dispersion, nonlinear effects due to relativistic mass variation in the presence of strong laser pulses. A new variant of the Gautschi-type integrator for reducing the number of time steps is proposed as a fast solver for such nonlinear wave-equations. In order to reduce the number of spatial grid points, a physically motivated quasi-envelope approach (QEA) is introduced. The new method turns out to reduce the computational time significantly compared to the standard leap-frog scheme.
\end{abstract}

Key words: Nonlinear wave propagation, relativistic plasma dynamics, Gautschi-type integrator, quasi-envelope approach

PACS: 02.70.-c; 02.30.Jr; 05.45.Yv; 52.27.Ny

\section{Introduction}

Wave motion in nonlinear dispersive media, such as the propagation of high-inten2sity laser pulses in plasmas, is of great interest both for technical applications as well as the understanding of fundamental nonlinear phenomena. Because of the vast number of different physical effects that can occur during nonlinear laser-plasma interaction in a specific physical system, it is of course necessary to simplify the analytical model as much as possible, while still including the important effects.

\footnotetext{
* Corresponding author.

Email address: spatschek@thphy . uni-duesseldorf.de (K.H. Spatschek).
} 
But it is also necessary to tailor the numerical methods to the specific problem, using the physical insight gained from the analytics. This yields both efficient and accurate numerical tools to study the dynamics of nonlinear wave equations.

In this paper we are interested in the fast numerical simulation of nonlinear wave propagation in dispersive media. We chose for demonstration the propagation of a laser pulse from vacuum into a cold underdense plasma layer. This can of course be generalized to a stratified medium consisting of several such layers, as discussed for example in [1] for pulse compression. It has been shown (see [2] and references therein) that in such situations, when the oscillatory velocity of the electrons approaches the speed of light, the resulting variations in the electron mass produce a significant contribution to the refractive index which can become more important than that due to ponderomotive force effects. Many results, e.g. [3-5], have been worked out for wave motion in the weakly relativistic regime. During the past years, slow and fast solitons caused by relativistic mass variations [6-9] have been discussed. In many cases, a slowly varying envelope approximation has been used, that results in nonlinear Schrödinger-type equations. The latter, however, may not be appropriate in the cases of subcycle solitons as well as for strongly inhomogeneous systems, where one has to include vacuum-plasma boundaries.

In the case of very high laser intensities and steep gradients of the fields, the plasma does not reach a local thermodynamic equilibrium and the particle distribution is essentially non-Maxwellian even on small scales. In this case, one has to solve the detailed dynamics of the distribution function in phase space. For that, Vlasov as well as particle-in-cell (PIC) codes are in use [10-13]. Vlasov codes integrate the kinetic Vlasov equation on an Eulerian grid in phase space. In PIC simulations, the phase space is sampled by macro-particles. From the positions and momenta of these particles the source terms of the field equations, which are solved on a grid, are interpolated. Hybrid codes that use a fluid description for the ions and a kinetic description for the electrons have also been developed [14], as well as codes that include binary collions using Monte-Carlo techniques [15].

If intensities as well as gradients are only moderately high, a magneto-hydrodynamical description becomes possible, especially if one is more interested in the dynamics of the laser pulse than that of the plasma. A fluid description is appropriate if the plasma is either locally Maxwellian or cold (which means that ponderomotive effects are less important than relativistic mass effects).

The present physical model results in a nonlinear wave equation for the vector potential coupled to the response of the medium. From the numerical point of view the problem is challenging because of the oscillatory behavior of the solution in space and in time. For discretization in space, the method of choice is the pseudospectral method which approximates spatial derivatives by using the fast Fourier method. The standard time integration scheme is the leap-frog method, which has a number of desirable properties, e.g. symmetry and symplecticity [16]. However, for linear 
stability (i.e. bounded error propagation in linearized equations), the product of the step size and the largest frequency of the system must be restricted by the value 2 , and good energy conservation requires this product to be even smaller (typically around 1/2) [17, Chapter XIII]. In the recent papers [18, 19], methods with improved stability and accuracy properties have been proposed. These methods allow larger time steps than the leap-frog method. In fact, it can be shown that in certain cases the error is of second order in time, independent of the highest frequencies arising in the system.

In the present paper, we propose a new variant of the Gautschi-type integrator, see [20] and further developments in [19], for reducing the number of time steps when solving a nonlinear wave equation. In order to reduce the number of spatial grid points, we introduce a physically motivated quasi-envelope approach (QEA). The new method turns out to reduce the computational time significantly compared to the standard leap-frog scheme. We also compare the results to PIC simulations performed with the VLPL (virtual laser plasma lab) [21].

\section{Physical model}

The basic set of equations being used for computation, is

$$
\begin{aligned}
\frac{\partial^{2} a}{\partial z^{2}}-\frac{\partial^{2} a}{\partial t^{2}} & =Q \frac{\left(n_{0}+\delta n\right)}{\gamma} a \\
\frac{\partial^{2} E}{\partial t^{2}}+Q n_{0} E & =-n_{0} \frac{\partial \gamma}{\partial z}
\end{aligned}
$$

where $a$ is the normalized vector potential,

$$
\gamma^{2}=1+|a|^{2}
$$

and $E$ the longitudinal electric field which gives the density modulation

$$
\delta n=-\frac{\partial E}{\partial z} .
$$

$Q<1$ is a parameter and $n_{0}$ is the normalized density profile. For the latter we shall use either a step profile or a profile with a linear increase and decrease of density at the plasma boundary. In vacuum $n_{0} \equiv 0$, which implies $E \equiv 0$.

The physical solution is a pulse localized in space. Due to finite energy it is bounded in $L^{2}$.

The validity of the model is restricted to the following assumptions. The wave equation (1) for a circularly polarized high-frequency wave vector potential $\mathbf{A}_{\perp}$ 
(with normalized transverse vector potential $a$ ) propagating in $z$-direction is influenced by relativistic electron mass variation $(\gamma$-factor). The space-variation of the $\gamma$-factor causes density modulations $(\delta n)$ and plasma oscillations as described by (2). Ion motion is neglected for fast propagation. The parameter $Q \equiv n_{\text {ion }}^{\max } / n_{\mathrm{c}}$ (maximum ion background density over critical density) should be less than 1 for wave propagation in an underdense medium and greater than 1/4 to avoid Raman instability.

Scalar as well as vector potentials are measured in units $m c^{2} / e$, whereas $n_{\text {ion }}^{\max }$ is used as a unit for the density, velocities are measured in units of $c$, and momenta are normalized with $m c$. We denote $m_{e} \equiv m$ as the electron rest mass. The electromagnetic wave has a wave-number $\mathbf{k}_{0}$; the inverse of its magnitude $\left(k_{0}^{-1}\right)$ is being used as the unit length. Similarly, the inverse of the wave-frequency $\omega_{0}$ has been used as the time unit. We have $\omega_{0}=c k_{0}$ in vacuum. The critical density $n_{\mathrm{c}}$ is defined via $\omega_{0}^{2} \equiv 4 \pi n_{\mathrm{c}} e^{2} / m$.

A derivation of the model can be found in Appendix A.

\section{Numerical schemes}

\subsection{Spatial discretization}

Due to the finite energy assumption on the physical solution it is possible to consider periodic boundary conditions for the discretization as long as the simulation box is big enough and one takes care of reflected pulses at the boundaries. For long time simulations this can be combined with a moving window technique.

Semi-discretization in space is done by a pseudospectral method with $N$ Fourier modes on a space interval $z \in z_{0}+[-L, L]$. This leads to the following system of coupled ordinary differential equations in time (the prime denotes time-derivative):

$$
\begin{aligned}
a^{\prime \prime} & =-D^{2} a+g(a, \delta n), & g(a, \delta n) & =-Q\left(n_{0}+\delta n\right) \frac{1}{\gamma} a, \\
E^{\prime \prime} & =-\omega^{2} E+f(a), & f(a) & =-n_{0} i D \sqrt{1+|a|^{2}} .
\end{aligned}
$$

Here, $D^{2}=\mathcal{F}_{N}^{-1} \mathcal{D}^{2} \mathcal{F}_{N}$, where $\mathcal{F}_{N}$ is the discrete Fourier-transform operator, and

$$
\mathcal{D}=\frac{\pi}{L} \operatorname{diag}\left(-\frac{N}{2},-\frac{N}{2}+1, \ldots,-1,0,1, \ldots, \frac{N}{2}-1\right) .
$$

The $j$ th component of the vectors $a(t)$ and $E(t)$ are approximations to $a\left(z_{j}, t\right)$ and $E\left(z_{j}, t\right)$ at $z_{j}=z_{0}+j \frac{2 L}{N}$, and $\omega^{2}=Q n_{0}$. 


\subsection{Gautschi-type exponential integrator for time discretization}

We propose to solve these equations with a modification of a Gautschi-type exponential integrator [19]. This integrator is motivated as follows: By the variation-ofconstants formula, the exact solution of

$$
y^{\prime \prime}=-\Omega^{2} y+F(y)
$$

satisfies

$$
\begin{aligned}
y(t+\tau)= & 2 \cos (\tau \Omega) y(t)-y(t-\tau) \\
& +\int_{0}^{\tau} \Omega^{-1} \sin ((\tau-s) \Omega)(F(y(t+s))+F(y(t-s))) \mathrm{d} s .
\end{aligned}
$$

For a constant inhomogeneity $F$ this yields

$$
y(t+\tau)=2 y(t)-y(t-\tau)+\tau^{2} \psi(\tau \Omega)\left(-\Omega^{2} y(t)+F\right),
$$

where

$$
\psi(x)=2 \frac{1-\cos x}{x^{2}}
$$

In the general case, a numerical scheme is obtained by substituting a suitable approximation of $F(y(t \pm s))$ into (8). This leads to approximations $y_{k} \approx y\left(t_{k}\right), t_{k}=k \tau$, defined by

$$
y_{k+1}=2 y_{k}-y_{k-1}+\tau^{2} \psi(\tau \Omega)\left(-\Omega^{2} y_{k}+F_{k}\right) .
$$

The simplest choice, originally proposed by Gautschi [20], is to set $F_{k}=F\left(y_{k}\right)$. However, the convergence analysis in [19] shows that in order to obtain secondorder error bounds, which are independent of the product of the step size with the frequencies, it is necessary to evaluate the nonlinearity $F$ at a filtered position, i.e. $F_{k}=F\left(\phi(\tau \Omega) y_{k}\right)$. If this filter function is omitted, then large errors are expected in the case when the product of the step size $\tau$ with one of the frequencies of the problem (the eigenvalues of $\Omega$ ) is an integer multiple of $\pi$. The filter function $\phi$ is a suitably chosen real function whose purpose is to filter out resonant frequencies, e.g.

$$
\phi(x)=\left(\frac{\sin x}{x}\right)^{2}, \quad \text { or } \phi(x)=\left(\frac{\sin x}{x}\right)^{2}\left(1+\frac{1}{2}(1-\cos x)\right) .
$$

The integrator applied to (7) then reads

$$
y_{k+1}=2 y_{k}-y_{k-1}+\tau^{2} \psi(\tau \Omega)\left(-\Omega^{2} y_{k}+F_{k}\right), \quad F_{k}=F\left(\phi(\tau \Omega) y_{k}\right) .
$$

In addition it is also possible to obtain approximations to the "velocities" $y^{\prime}$ via

$$
y_{k+1}^{\prime}=y_{k-1}^{\prime}+2 \tau \sigma(\tau \Omega)\left(-\Omega^{2} y_{k}+F_{k}\right),
$$

where $\sigma(x)=\sin x / x$. Note that approximating the "velocities" by standard finite differences will lead to inaccurate results due to the oscillatory behavior of $y$. 
For $\Omega=0$ the Gautschi-type integrator reduces to the well known leap-frog or Störmer-Verlet method. We will use (9) and, if desired, (10) for the integration of (5) for the vector potential.

The accuracy of the integrator may be further improved if approximations to the inhomogeneity are available at additional times. This is only true if we solve the equations (6) for the electrical field because there the inhomogeneity only depends on $a$. If we solve the equation for $a$ first, we have approximations $a_{j} \approx a\left(t_{j}\right)$ for $j=k-1, k$, and $k+1$. We then replace $f(a)$ by an interpolation polynomial of degree two interpolating in $\left(t_{k-1}, f\left(a_{k-1}\right)\right),\left(t_{k}, f\left(a_{k}\right)\right)$, and $\left(t_{k+1}, f\left(a_{k+1}\right)\right)$. Note that we consider the circular polarized case, in which $f$ is a smooth function. Using this interpolation polynomial instead of $F(y(t \pm s))$ in (8) yields

$$
\begin{aligned}
E_{k+1}=2 E_{k}-E_{k-1} & +\tau^{2} \psi(\tau \omega)\left(-\omega^{2} E_{k}+f\left(a_{k}\right)\right) \\
& +\tau^{4} \chi(\tau \omega)\left(f\left(a_{k+1}\right)-2 f\left(a_{k}\right)+f\left(a_{k-1}\right)\right)
\end{aligned}
$$

for (6), where

$$
E_{k} \approx E\left(t_{k}\right) \quad \text { and } \quad \chi(x)=2 \frac{\cos x-1+\frac{1}{2} x^{2}}{x^{4}} .
$$

The scheme (11) is of order four, if $a_{j}, j=k-1, k, k+1$ are exact or sufficiently accurate approximations of $a\left(t_{j}\right)$. However, the coupled scheme (9), (11) cannot be better than second order.

\subsection{Choice of operators}

For solving (5) the obvious choice would be using (9) with $\Omega=D$. By construction, the Gautschi-type integrator then solves equations $y^{\prime \prime}=-\Omega^{2} y+F$ with constant $F$ exactly. Due to the special form of the nonlinearity $g$, we can enlarge the part which is integrated exactly by writing

$$
g(a, \delta n)=-\alpha a+\widetilde{g}(a, \delta n)
$$

and setting $\Omega^{2}=D^{2}+\alpha$ for a suitable $\alpha$. If the pulse is inside the plasma, the dominant term of $g$ is linear in $a$, which suggests to choose $\alpha=Q$. Outside the plasma (where $n_{0}=0$ ) the nonlinearity is negligible so that one should set $\alpha=0$.

\subsection{Quasi-envelope approach}

The motivation behind the quasi-envelope approach (QEA) is illustrated on a numerical result shown in Fig. 1: the spectrum of the vector potential splits into two 


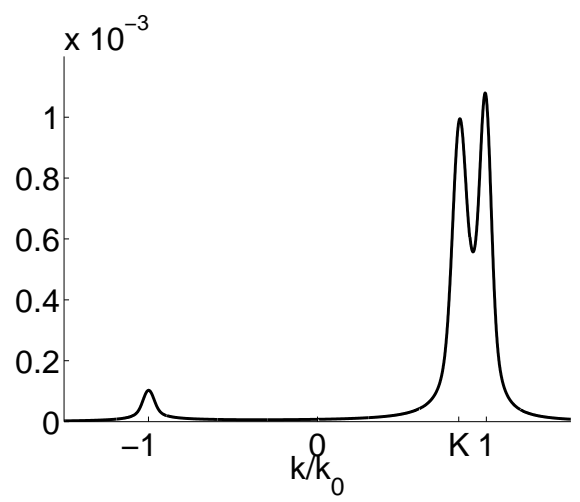

Fig. 1. Spectrum of the vector potential while entering the plasma, $K=\sqrt{1-Q}$.

parts. The important part is concentrated around a certain characteristic wave number depending on whether the pulse propagates inside or outside of the plasma. In addition there is another peak resulting from reflection which is not of interest in our physical application. Therefore, it is sufficient to resolve the main pulse only. The number of spatial grid points required can be reduced significantly by shifting the spectrum appropriately, i.e. we replace the vector potential $a$ by

$$
a(z, t)=\widetilde{a}(z, t) e^{i \kappa z}
$$

and solve (1) for $\widetilde{a}$ instead of $a$. This yields

$$
\frac{\partial^{2}}{\partial t^{2}} \widetilde{a}=\frac{\partial^{2}}{\partial z^{2}} \widetilde{a}+2 i \kappa \frac{\partial}{\partial z} \widetilde{a}-\kappa^{2} \widetilde{a}-Q\left(n_{0}+\delta n\right) \frac{1}{\gamma} \widetilde{a} \quad, \quad \gamma^{2}=1+|\widetilde{a}|^{2} .
$$

Note that in the "classical" envelope approximation $\partial^{2} \tilde{a} / \partial z^{2}$ is neglected, leading to a Schrödinger type equation in $z$. In the spatially discretized equation $(5), \mathcal{D}^{2}$ has to be replaced by $(\mathcal{D}+\kappa)^{2}$. The value of $\kappa$ can be varied for different positions of the pulse (inside/outside of the plasma or at the boundary), so we choose $\kappa=\sqrt{1-Q}$, $\kappa=1$ or the mean value of both.

\subsection{Multilevel approach}

Obviously, the spatial grid size is determined by the necessity of resolving reflections arising at jumps of the plasma density. If we have a sharp jump (for instance in the case of a rectangular density profile shown in Fig. 2), then the reflections require small spatial grid sizes only when the pulse enters or leaves the plasma. This can be exploited in a standard way by using two (or more) different grids. In our case we used a fine grid in transitions between vacuum and plasma and a coarse one in the remaining simulation. Switching between coarse and fine grid is done by interpolation and from the fine to the coarse grid by restriction (both in Fourier space). Note that this switch requires to recompute the differential operator and hence the matrix operators required for the Gautschi-type integrator. 


\subsection{Overall numerical method}

We suggest to combine the strategies described above. This requires the computation of three or more sets of operators: one in vacuum $\left(\alpha_{\mathrm{v}}=0, \kappa_{\mathrm{v}}=1\right.$, coarse grid), one in plasma $\left(\alpha_{\mathrm{p}}=Q, \kappa_{\mathrm{p}}=\sqrt{1-Q}\right.$, coarse grid), and one in the transition $\left(\alpha_{\mathrm{t}}=Q / 2, \kappa_{\mathrm{t}}=(1+\sqrt{1-Q}) / 2\right.$, fine grid $)$, and possibly additional sets if the pulse gets too steep to be resolved on the coarse grid in plasma due to nonlinear pulse compression. If background density is small (so that the difference between vacuum and plasma wavelength is also small) and the density profile has no sharp jump (so that no reflection occurs), it maybe sufficient to use the same set of operators for both the transitional region and the plasma region on the same coarse grid, with a $\kappa$ halfway between vacuum and plasma wave-number. Recall that in vacuum, there is no nonlinearity, and thus the Gautschi-type integrator solves the problem exactly for arbitrary time steps. Obviously, it is not necessary to compute filter functions in this case.

\section{Exemplary results}

\subsection{Description of the simulated problem}

For runtime comparison we chose a simulation box of length $1000 \lambda$. As density profile we used a piecewise linear function which is 0 for $z$ smaller than $100 \lambda$ and greater than $810 \lambda, 1$ for $105 \lambda<z<805 \lambda$ and linear in between. In this case, the multilevel approach is not necessary, because nearly no reflection occurs at the plasma boundaries. To simplify the simulational setup for the runtime comparison further, for methods with QEA, only one set of operators is used with a mean value of vacuum and plasma wavelength. With an additional set of operators for the plasma part, the results discussed below would be even better. But for a low background density like $Q=0.3$, which we used, the results are already very good. For denser plasmas (e.g. $Q=0.6$ ), switching of operators between plasma boundary and plasma parts of the density profile becomes a necessity. For the multilevel tests we used a rectangular density profile beginning at $105 \lambda$ and ending at $805 \lambda$, cf. Fig. 2, and we included the different operators discussed in Sec. 3.6.

The initial conditions for the vector potential in vacuum were calculated from

$$
a(z, t)=a_{0} e^{-\frac{\left(z-z_{0}-t\right)^{2}}{W_{0}^{2}}} e^{i(z-t)}
$$

at $t=0$ and $t=\tau$. The parameters were $z_{0}=35 \lambda, W_{0}=10 \lambda$, and $a_{0}=0.1$ or $a_{0}=0.12$. Due to our normalization $\lambda=2 \pi$. 

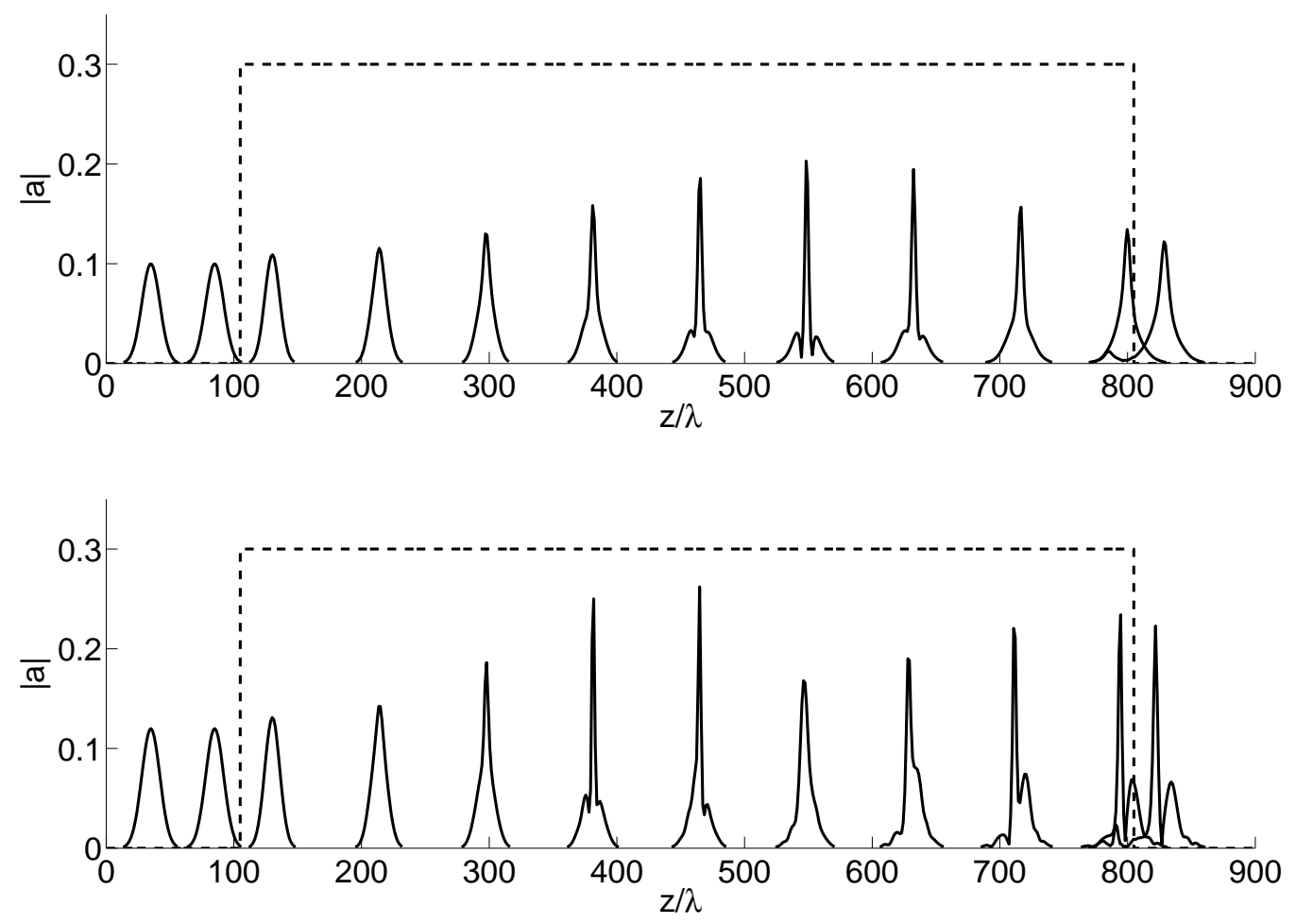

Fig. 2. Pulse propagation (solid line) shown at different times and background density profile (dashed line) for $a_{0}=0.1$ (top) and $a_{0}=0.12$ (bottom).

As an example, the time evolution for two different initial amplitudes (with the rectangular plasma profile) is shown in Fig. 2. In both cases, first a compression and then a widening of the pulse can be seen. If we calculate the amplitude for the single soliton state of the Schrödinger model for a $W_{0}=10 \lambda$ wide $\operatorname{sech}\left(z / W_{0}\right)$ pulse (see [1]), we get $a_{0} \approx 0.038$. A simulation of such a pulse verifies that the soliton state of our model equations is close to this. For the two amplitudes above, this implies that we are well within the nonlinear regime. It also suggests that the initial condition with $a_{0}=0.1$ is close to a bound two-soliton state, while for $a_{0}=0.12$ it is clearly above. In the latter case the pulse compresses more and earlier, and more energy is radiated away from the core of the pulse after the compression.

As benchmarks for the accuracy of the different numerical schemes, we used two error measures. Since we do not have an analytical solution of the nonlinear model equations, we computed a reference solution on a very fine grid $\left(N=2^{17}\right)$ with very small time steps. We then used it to measure the error in maximum amplitude squared (amplitude error) and its position (phase error) at different times of the simulation results. Since the simulations were computed on coarser grids (especially the QEA solutions) we first Fourier interpolated to the same number of grid points as the reference solution. 

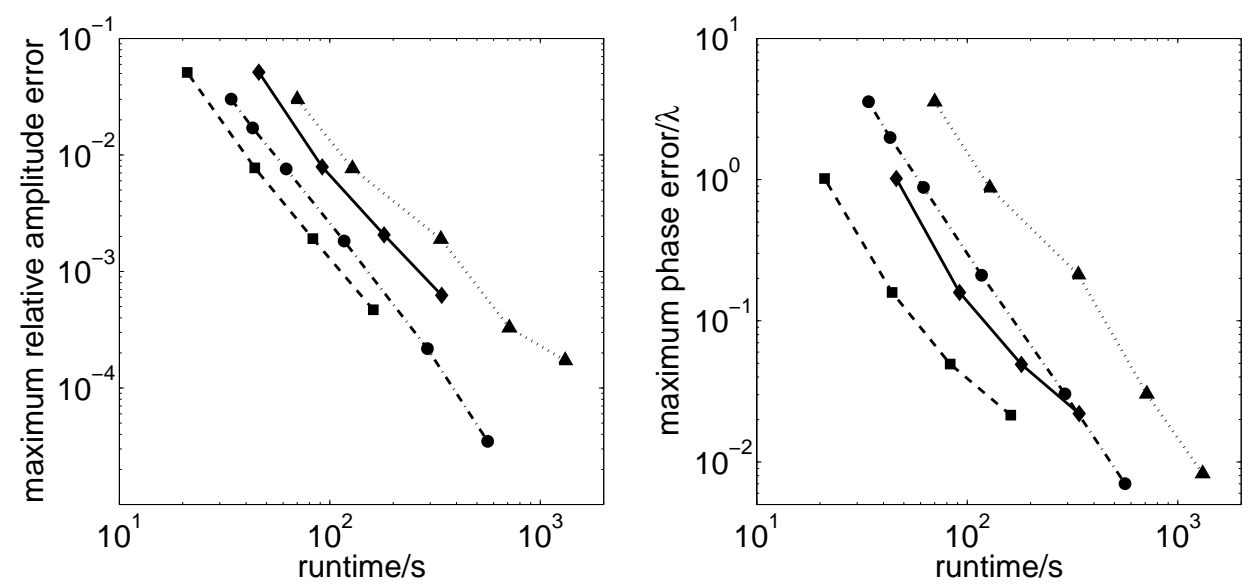

Fig. 3. Maximum amplitude and phase error vs. runtime $\left(a_{0}=0.1\right)$ for varying $\tau$ for leap-frog (dotted+triangles), Gautschi (solid+diamonds), leap-frog + QEA (dash-dotted+circles) and Gautschi + QEA (dashed+squares). We used $N=2^{12}$ for methods without QEA and $N=2^{11}$ for methods with QEA (see also Table 1).
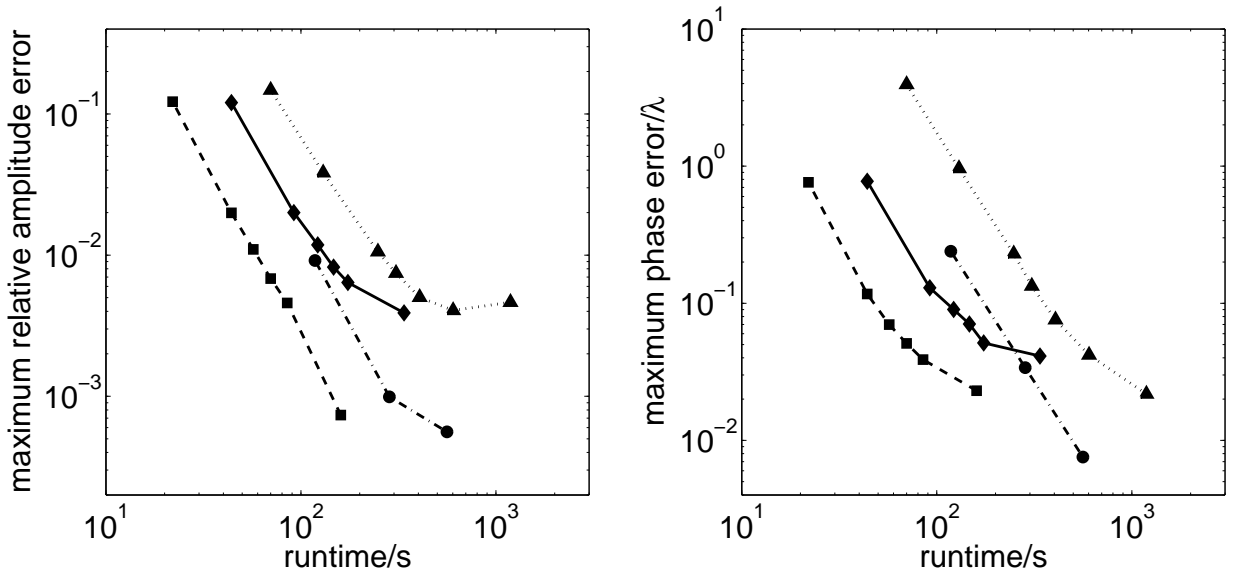

Fig. 4. Same as Fig. 3, but for $a_{0}=0.12$.

\subsection{Effect of different time-integration schemes}

If the vector potential is held in Fourier space and only transformed back for the evaluation of the nonlinearity/inhomogeneity, one has to compute six fast Fourier transforms per time step for the leap-frog method (two for the nonlinearity of the wave equation, two for the inhomogeneity of the plasma response, and two for the transformation of $E$ ). There is one more Fourier transform needed for the Gautschitype integrator since in each step the filtered as well as the nonfiltered vector potential is required in real space. In addition, one has to compute the products with the matrix functions $\psi, \phi$, and possibly $\sigma$. Obviously computing a single time step with the Gautschi-type integrator is more expensive than one time step with the leapfrog method. But it turns out that the Gautschi-type method allows larger time steps in order to reach the same accuracy. 


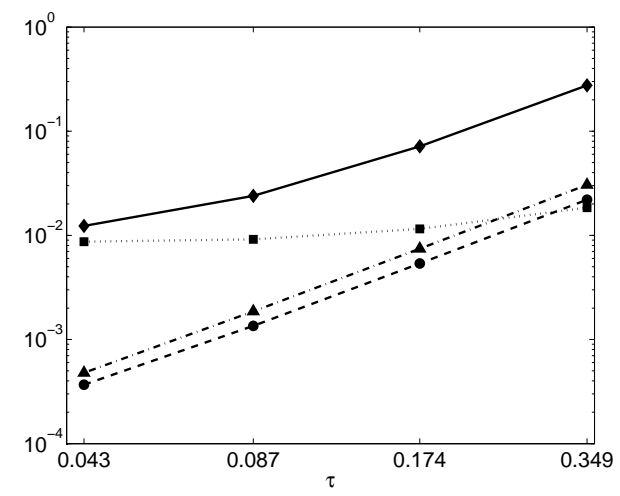

Fig. 5. Amplitude and phase error plotted over the time step $\tau$ for the Gautschi-type integrator including quasi-envelope approach with and without the variant described in Section 3.3. Phase/amplitude error with $\alpha=0$ (solid/dashed line) and $\alpha=Q$ within the plasma (dotted/dash-dotted line) for $a_{0}=0.1$.

In Fig. 3 and Fig. 4 maximum relative amplitude error (left) and maximum phase error in $\lambda$ (right) are plotted over computational time. Each curve represents one integrator on one spatial grid with different time steps.

For a given tolerance for the relative amplitude error the leap-frog method (dotted+triangles) needs two times smaller time steps than the Gautschi-type integrator (solid+diamonds) on the same spatial grid $\left(N=2^{12}\right)$. In our examples this reduces the computational time by a factor of 1.5 (see Table 1 ). If the phase error is taken into account, too, the gain in computational time is even greater.

\subsection{Effect of choice of operators}

The effect of the choice of operators is illustrated in Fig. 5 for the case $a_{0}=0.1$. It is observed that the choice of $\alpha=Q$ within the plasma reduces the phase error significantly while the error in the amplitude is only slightly larger. However, for $a_{0}=0.12$ switching between the operators did not pay off. The reason for this might be the increased density variation compared to the smaller amplitude. The results in Fig. 5 were computed including QEA of Section 3.4, but the method showed the same behavior when combined with other variants described above. The phase error is given in terms of $\lambda$ whereas the amplitude error is given relatively compared to the reference amplitude. In both cases the error is averaged over pulses at 100 different positions spread evenly over the computation interval.

\subsection{Effect of quasi-envelope approach}

By applying the quasi-envelope approach to the leap-frog method as well as the Gautschi-type integrator, the number of spatial grid points can be significantly re- 
Table 1

\begin{tabular}{l|c|c|c|c|c|c}
\multirow{2}{*}{} & \multicolumn{3}{|c|}{$a_{0}=0.1$} & \multicolumn{3}{c}{$a_{0}=0.12$} \\
\cline { 2 - 7 } & $N$ & $\tau$ & time/min. & $N$ & $\tau$ & time/min. \\
\hline LF & $2^{12}$ & 0.1 & $2: 10$ & $2^{12}$ & 0.04 & $5: 07$ \\
\hline LF + QEA & $2^{11}$ & 0.1 & $1: 03$ & $2^{11}$ & 0.05 & $1: 57$ \\
\hline Gautschi & $2^{12}$ & 0.2 & $1: 32$ & $2^{12}$ & 0.12 & $2: 28$ \\
\hline Gautschi + QEA & $2^{11}$ & 0.2 & $0: 44$ & $2^{11}$ & 0.12 & $1: 10$
\end{tabular}

Runtimes for maximum one percent relative amplitude error. $N$ is the number of spatial grid points, $\tau$ is the time step size. Computational details: Pentium 4, 3.0 GHz, Intel C++ 8.1, FFT routines from Intel Math Kernel Library 7.2.

duced without loss of accuracy (see curves with and without QEA in Fig. 3 and 4). Since the major part of computational time is spent on fast Fourier transforms, which cost $O(N \log N)$ operations, the reduction of grid points by a factor of 2 again leads to a saving in computational time of more than a factor of 2 . Another reason for a more than linear reduction in computational time is that smaller arrays are more likely to fit into the cache of the processor. For small enough arrays, a whole time step can run from CPU cache. We observed that QEA is more effective in reducing the amplitude error, while the Gautschi-type method is more effective in reducing the phase error.

The parameters for the discretization needed to achieve a maximum relative amplitude error of $10^{-2}$ are summarized in Table 1. Exemplary runtimes for one specific hardware/software setup are also given.

If one compares the standard leap-frog method to the new variant of the Gautschitype integrator combined with QEA, the computational time is reduced by a factor of 3 in the first and even by a factor of 4.5 in the second example. If we set a bound lower than $10^{-2}$ for the amplitude error, we see that without QEA this error bound cannot be reached by only reducing $\tau$. This is because the error due to the coarse spatial resolution limits the accuracy that can be reached. Thus a finer grid is needed, which results in a corresponding increase of computational time, while the discretization for QEA can stay the same (see Fig. 6).

\subsection{Effect of two-level approach}

The benefit of the two-level approach suggested in Section 3.5 is illustrated in Fig. 7. The reference solution as well as the simulation results are shown at $t=$ $700 \cdot 2 \pi$ for a plasma jump and $a_{0}=0.12$. It can be seen that in this case it is possible to work on a coarse grid $\left(N=2^{11}\right)$ in the major part of the simulation but it is not possible to do the whole simulation on the coarse grid. In the transition we interpolated to $2^{13}$ grid points. 


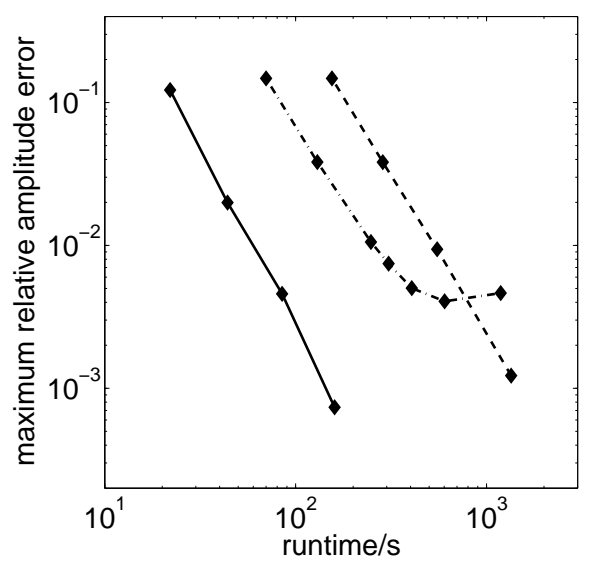

Fig. 6. Maximum amplitude error vs. runtime ( $a_{0}=0.12$ ) for constant $N$ and varying $\tau$ for leap-frog with $N=2^{13}$ (dashed), leap-frog with $N=2^{12}$ (dash-dotted) and Gautschi + QEA with $N=2^{11}$ (solid).

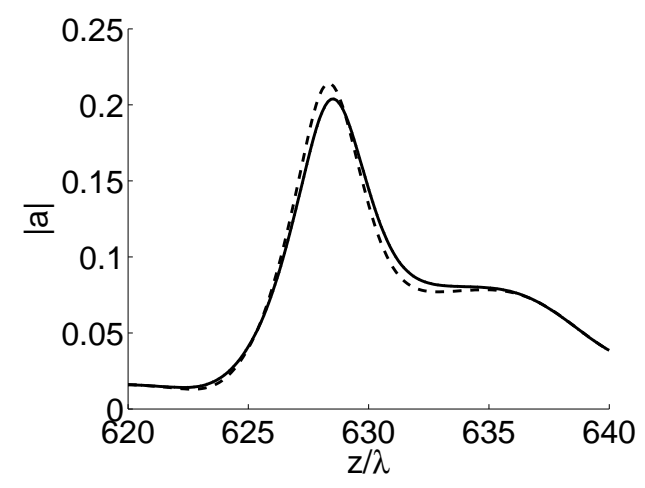

Fig. 7. Results of simulations using the two-level approach compared to a one-level simulation on the (same) coarse grid only. Solid: reference solution, dashed: solution computed on a coarse grid only, dotted: two-level approach (curve coincides with the solid one).

\subsection{Comparison with PIC}

Finally, we compare with PIC simulations performed with VLPL [21]. Since PIC simulates $\mathbf{E}$ and $\mathbf{B}$ instead of $\mathbf{A}$, we base our comparison on intensities, calculated by

$$
I=\frac{1}{2}\left(|\mathbf{E}|^{2}+|\mathbf{B}|^{2}\right)=\frac{1}{2}\left(\left|\frac{\partial}{\partial t} \mathbf{A}\right|^{2}+\left|\frac{\partial}{\partial z} \mathbf{A}\right|^{2}\right) .
$$

For the Gautschi-type method, one has to use (10) for the time-derivative, and for QEA $\partial / \partial z \rightarrow \partial / \partial z+i \kappa$. The difference in amplitudes between the reference solution for the reduced model and PIC (see Fig. 8) and the Gautschi-type method with QEA for the parameters given in Table 1 are of the same order. This implies that, even with a relatively coarse discretization, the error of the simulations with our fastest solver is within the accuracy of the reduced model, which seems to be 

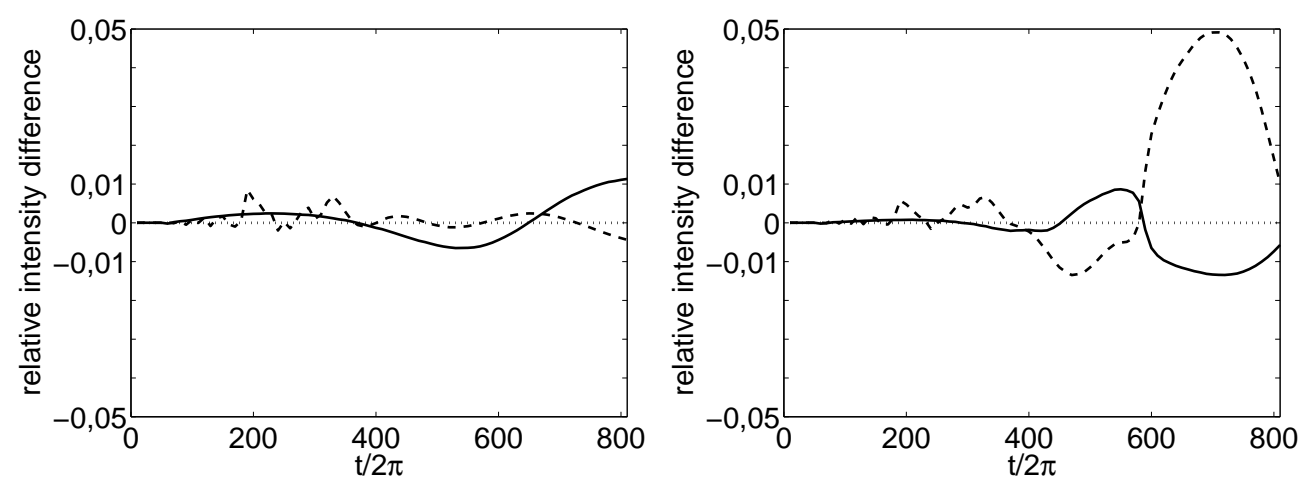

Fig. 8. Relative difference in intensity to the reference solution of the reduced model for $a_{0}=0.1$ (left) and $a_{0}=0.12$ (right). Gautschi+QEA (see Table 1, solid) and PIC (dashed) with $N=2 \cdot 10^{5}, \tau=d z(N)$ and 3 particles per cell, runtime around $5: 30 h$.

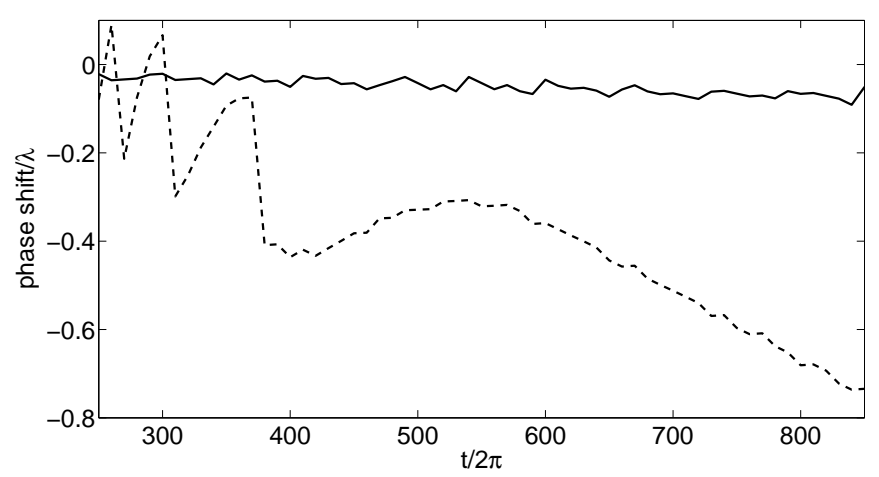

Fig. 9. Phase-difference to the exact linear solution for PIC (dashed) and Gautschi+QEA (solid), both with $a_{0}=0.0001$.

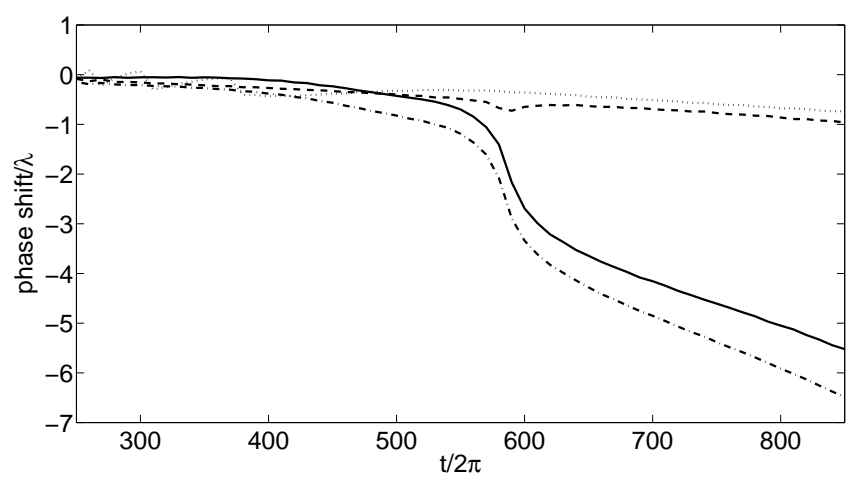

Fig. 10. Phase-difference to the exact linear solution for PIC ( $a_{0}=0.12$ : dash-dotted and $a_{0}=0.0001$ : dotted $)$ and Gautschi+QEA $\left(a_{0}=0.12\right.$, solid $)$, difference between PIC and Gautschi+QEA for $a_{0}=0.12$ (dashed).

at the border of applicability at $a_{0}=0.12$.

We also noticed, that there is a systematic difference in group velocity between PIC solutions and ours. To understand whether this is due to numerical error in 
PIC and/or our solvers, we made simulations with both for a very small amplitude $\left(a_{0}=0.0001\right)$. The combination of small amplitude and a cold plasma allows to test the phase error of the numerical simulations against the known linear analytical solution. The results in Fig. 9 show that PIC (dashed) produces a slight error in group velocity even on a fine grid, whereas Gautschi+QEA (solid) with coarse discretization is close to the exact solution.

In Fig. 10 we compare the phase shift (with respect to the exact linear solution) of VLPL (dash-dotted) and the Gautschi+QEA simulation from Table 1 (solid) in the nonlinear case $\left(a_{0}=0.12\right)$. The difference between the two (dashed) is consistent with the linear phase error of PIC (dotted). This shows that the difference in phase between nonlinear PIC and Gautschi+QEA is mostly linear phase error of PIC, which could also influence the accuracy of the amplitude calculation.

\section{Conclusion}

In the present paper, we have investigated numerically a physical model for wave propagation in a nonlinear, dispersive medium. The model applies to strong laser pulse propagation into a relativistic plasma. It consists of two coupled equations for the high-frequency laser field and the low-frequency plasma response. The latter causes, besides dispersion, nonlinear effects due to relativistic mass variation. A fast numerical solver for the coupled equations is presented. It incorporates two main ideas. First, a new variant of the Gautschi-type integrator for reducing the number of time steps is proposed. Secondly, in order to reduce the number of spatial grid points, a physically motivated quasi-envelope approach (QEA) is introduced. The new method turns out to reduce the computational time significantly (for example by a factor of 5 even for low accuracy demands) compared to the standard leap-frog scheme. We also found that for the weakly nonlinear regime the combination of the reduced model and our fast solver is as accurate as a first-principle PIC simulation. The latter, however, needs much more time.

The advantages of the present scheme for oscillatory problems are discussed in detail. It can be expected that the main ideas will also be useful for other physical problems being highly oscillatory in nature, eg. the non-linearized model. We currently investigate the extension to a three dimensional geometrie.

\section{Acknowledgment}

We would like to thank A. Pukhov and O. Shorokov for providing the VLPL PICcode and helpful discussions. 
This work has been supported by the Deutsche Forschungsgemeinschaft through the Transregio-SFB 18.

\section{A Details of the physical model}

From Maxwell's equations we get for the vector potential $\mathbf{A}$ and scalar potential $\varphi$ in Coulomb gauge

$$
-\nabla^{2} \mathbf{A}+\frac{1}{c^{2}} \frac{\partial^{2} \mathbf{A}}{\partial t^{2}}=\frac{1}{c} \frac{\partial \nabla \varphi}{\partial t}+\left(\frac{4 \pi}{c}\right) \mathbf{j}
$$

For fixed ions (we are considering fast solitary waves) the current density is

$$
\mathbf{j}=-e n_{e} \mathbf{v}_{e}
$$

By electron velocity and density, the wave equation is coupled to the continuity equation for the electron density

$$
\frac{\partial}{\partial t} n_{e}+\nabla \cdot\left(n_{e} \mathbf{v}_{e}\right)=0
$$

and the electron momentum balance

$$
\left(\frac{\partial}{\partial t}+\mathbf{v}_{e} \cdot \nabla\right) \mathbf{P}_{e}=e\left[-\nabla \varphi-\frac{1}{c} \frac{\partial \mathbf{A}}{\partial t}+\frac{1}{c} \mathbf{v}_{e} \times(\nabla \times \mathbf{A})\right]
$$

which describe the nonlinear response of the medium. We can thus identify two sources of nonlinearity, density oscillation and the relativistic mass factor, because

$$
\mathbf{v}_{e}=\frac{\mathbf{P}_{e}}{m_{e} \gamma_{e}}
$$

with

$$
\gamma_{e}=\frac{1}{\sqrt{1-\left(\frac{\mathbf{v}_{e}}{c}\right)^{2}}}=\sqrt{1+\left(\frac{\mathbf{P}_{e}}{m_{e} c}\right)^{2}} .
$$

By some straightforward manipulations, we can write the momentum balance in the form

$$
\frac{\partial}{\partial t}\left(\mathbf{P}_{e}-\frac{e}{c} \mathbf{A}\right)=e \nabla \varphi-m c^{2} \nabla \gamma_{e}+\frac{1}{m_{e} \gamma_{e}} \mathbf{P}_{e} \times\left[\nabla \times\left(\mathbf{P}_{e}-\frac{e}{c} \mathbf{A}\right)\right] .
$$

To normalize the equations we use the inverse wave-number $\left(k_{0}^{-1}\right)$ and inverse wave-frequency $\omega_{0}^{-1}$ of the laser carrier as unit length and time $\left(\omega_{0}=c k_{0}\right.$ in vac- 
uum). A wave can propagate in a medium with (constant) electron density $n_{e}$ provided $\omega_{0}>\omega_{p}$ where

$$
\omega_{p}=\sqrt{\frac{4 \pi n_{e} e^{2}}{m_{e}}}
$$

is the electron plasma frequency. We use the maximum ion background density $n_{\text {ion }}^{\max }$ as the density unit. The critical density for wave propagation $n_{\mathrm{c}}$ follows from the condition

$$
\frac{4 \pi n_{\mathrm{c}} e^{2}}{m_{e}}=\omega_{0}^{2}
$$

Thus, the parameter

$$
Q=\frac{n_{\text {ion }}^{\max }}{n_{\mathrm{c}}}
$$

can be introduced; it has to be less than 1 for wave propagation.

Scalar as well as vector potentials are measured in units of $m_{e} c^{2} / e$. Velocities are measured in units of $c$, and momenta are normalized with $m_{e} c$. From now on, we omit the index $e$ for electrons. The electron momentum balance then is

$$
\frac{\partial}{\partial t}(\mathbf{P}-\mathbf{A})-\frac{\mathbf{P}}{\gamma} \times \nabla \times(\mathbf{P}-\mathbf{A})=\nabla(\varphi-\gamma) .
$$

Let us now write the basic equations under the assumption that the wave propagates in $z$-direction, so that all variables depend only on one space coordinate, i.e.

$$
\mathbf{A}=\mathbf{A}(z), \quad n=n(z), \quad \varphi=\varphi(z), \quad P_{z} \rightarrow P .
$$

From the Coulomb gauge follows the purely transverse nature of the wave $(\mathbf{A}=$ $\left.\mathbf{A}_{\perp}\right)$. The wave equation for the transverse component $\mathbf{A}_{\perp}$ now reads

$$
\frac{\partial^{2}}{\partial z^{2}} \mathbf{A}_{\perp}-\frac{\partial^{2}}{\partial t^{2}} \mathbf{A}_{\perp}=Q n \frac{\mathbf{P}_{\perp}}{\gamma}
$$

The longitudinal part of the wave equation simplifies to

$$
\frac{\partial^{2} \varphi}{\partial t \partial z}+Q n \frac{P}{\gamma}=0
$$

Within the plasma, the perpendicular electron momentum balance

$$
\frac{\partial}{\partial t}\left(\mathbf{P}_{\perp}-\mathbf{A}_{\perp}\right)+\left(\frac{P}{\gamma}\right) \frac{\partial\left(\mathbf{P}_{\perp}-\mathbf{A}_{\perp}\right)}{\partial z}=0
$$

has the special solution

$$
\mathbf{P}_{\perp}=\mathbf{A}_{\perp},
$$

which also simplifies the longitudinal electron momentum balance

$$
\frac{\partial P}{\partial t}-\mathbf{P}_{\perp} \cdot \frac{\partial\left(\mathbf{P}_{\perp}-\mathbf{A}_{\perp}\right)}{\partial z}=\frac{\partial(\varphi-\gamma)}{\partial z} .
$$


This leads to the basic set of equations:

$$
\begin{aligned}
\frac{\partial^{2}}{\partial z^{2}} \mathbf{A}_{\perp}-\frac{\partial^{2}}{\partial t^{2}} \mathbf{A}_{\perp} & =Q n \frac{\mathbf{A}_{\perp}}{\gamma} \\
\frac{\partial^{2} \varphi}{\partial t \partial z}+Q n \frac{P}{\gamma} & =0 \\
\frac{\partial^{2} \varphi}{\partial z^{2}} & =Q\left(n-n_{0}\right) \\
\frac{\partial n}{\partial t}+\frac{\partial}{\partial z}\left(\frac{n P}{\gamma}\right) & =0 \\
\frac{\partial P}{\partial t} & =\frac{\partial(\varphi-\gamma)}{\partial z}
\end{aligned}
$$

where $n_{0}$ is the time-independent part of $n$ (which is identical to the fixed ion background), while in the following $\delta n$ denotes the first order density oscillation.

Next, we specify to circular polarized waves, introducing

$$
a=A_{x}+i A_{y}
$$

This yields

$$
\gamma^{2}=1+|a|^{2}+P^{2}
$$

Writing

$$
n=n_{0}+\delta n=n_{0}+\frac{1}{Q} \frac{\partial^{2} \varphi}{\partial z^{2}} \equiv n_{0}-\frac{1}{Q} \frac{\partial \widetilde{E}}{\partial z},
$$

the wave equation has the form

$$
\frac{\partial^{2} a}{\partial z^{2}}-\frac{\partial^{2} a}{\partial t^{2}}=Q \frac{\left(n_{0}+\delta n\right)}{\gamma} a
$$

The plasma response is determined via the equations

$$
\begin{aligned}
\frac{\partial \delta n}{\partial t} & =-\frac{\partial}{\partial z}\left[\frac{\left(n_{0}+\delta n\right) P}{\gamma}\right] \\
\frac{\partial P}{\partial t} & =-\widetilde{E}-\frac{\partial \gamma}{\partial z} \\
\frac{\partial \widetilde{E}}{\partial t} & =Q \frac{\left(n_{0}+\delta n\right) P}{\gamma}
\end{aligned}
$$

Note that the additional equation

$$
\frac{1}{Q} \frac{\partial \widetilde{E}}{\partial z} \equiv \frac{\partial E}{\partial z}=-\delta n
$$

is the solvability condition of (A.29) and (A.27). 
We may simplify the plasma response by taking a time derivative of (A.29) and substituting the corresponding expressions from (A.28) and (A.27). The final result is

$$
\frac{\partial^{2} \widetilde{E}}{\partial t^{2}}+Q n_{0} \widetilde{E}=-Q n_{0} \frac{\partial \gamma}{\partial z}+R
$$

where

$$
R:=-Q n_{0} \frac{\partial}{\partial t}\left[\left(1-\frac{1}{\gamma}\right) P\right]+Q n_{0} \frac{\partial}{\partial t}\left(\delta n \frac{P}{\gamma}\right)
$$

In the main text we assume $R \approx 0$. The reason is $R \sim O\left(\varepsilon^{4}\right)$, whereas the other terms in Eq. (A.31) are of order $\varepsilon^{2}$. Here, $\varepsilon$ characterizes the order of magnitude of $a$. Then the balance (A.28) suggests the ordering $\delta n \sim P \sim a^{2} \sim O\left(\varepsilon^{2}\right)$. Note that we do not scale the space- and time-variables. From (A.32) we then find $R \sim O\left(\varepsilon^{4}\right)$.

By similar arguments, to leading order, we can set $P \approx 0$ in (A.24) such that

$$
\gamma^{2}=1+|a|^{2}
$$

Together with (A.30) the basic set of equations is

$$
\begin{aligned}
\frac{\partial^{2} a}{\partial z^{2}}-\frac{\partial^{2} a}{\partial t^{2}} & =Q \frac{\left(n_{0}+\delta n\right)}{\gamma} a \\
\frac{\partial^{2} E}{\partial t^{2}}+Q n_{0} E & =-n_{0} \frac{\partial \gamma}{\partial z}
\end{aligned}
$$

The last equation may also be written in the form

$$
\frac{\partial^{2} \delta n}{\partial t^{2}}+Q n_{0} \delta n=n_{0} \frac{\partial^{2} \gamma}{\partial z^{2}}
$$

if the density profile $n_{0}$ is piecewise constant or depends only very slowly on $z$.

In the vacuum part of the density profile, where $n_{0}=0$, equations (A.34) and (A.35) reduce to

$$
\frac{\partial^{2} a}{\partial z^{2}}-\frac{\partial^{2} a}{\partial t^{2}}=0
$$

\section{References}

[1] O. Shorokov, A. Pukhov, I. Kostyukov, Self-compression of laser pulses in plasma, Phys. Rev. Lett. 91 (2003) 265002-1-4.

[2] S. Atzeni, J. Meyer-Ter-Vehn, The Physics of Inertial Fusion: BeamPlasma Interaction, Hydrodynamics, Hot Dense Matter (International Series of Monographs on Physics, Oxford U.P., Oxford, 2004.

[3] K. Spatschek, Self-focusing of electromagnetic waves as a result of relativistic electron-mass variation, J. Plasma Phys. 18 (1977) 293-303. 
[4] K. Spatschek, Relativistic effects on electron plasma waves, Phys. Rev. A 16 (1977) 2470-2472.

[5] M. Yu, P. Shukla, K. Spatschek, Localization of high-power laser pulses in plsmas, Phys. Rev. A 18 (1978) 1591-1596.

[6] P. Kaw, A. Sen, T. Katsouleas, Self-compression of laser pulses in plasma, Phys. Rev. Lett. 68 (1992) 3172-3175.

[7] S. Bulanov, F. Califano, T. Esirkepov, K. Mima, N. Naumova, K. Nishihara, F. Pegoraro, Y. Sentoku, V. Vshivkov, Generation of subcycle relativistic solitons by superintense laser pulses in plasmas, Physica D 152-153 (2001) 682-693.

[8] D. Farina, S. Bulanov, Relativistic electromagnetic solitons in the electron-ion plasma, Phys. Rev. Lett. 86 (2001) 5289-5292.

[9] L. Hadzievski, M. Jovanovic, M. Skoric, K. Mima, Stability of one-dimensional electromagnetic solitons in relativistic laser plasmas, Phys. Plasmas 9 (2002) 25692574.

[10] C. Birdsall, A. Langdon, Plasma Physics via Computer simulation, Adam Hilger, New York, 1991.

[11] R. Hockney, J. Eastwood, Computer Simulation using Particles, Adam Hilger, New York, 1988.

[12] J. Dawson, Particle simulation of plasmas, Rev. Mod. Phys. 55 (1983) 403.

[13] J. Villasenor, O. Buneman, Rigorous charge conservation for local electromagnetic field solvers, Comp. Phys. Comm. 69 (1992) 306.

[14] D. Harned, Quasineutral hybrid simulation of macroscopic plasma phenomena, J. Comput. Phys. 47 (1982) 452.

[15] C. Birdsall, Particle-in-cell charged-particle simulations, plus Monte Carlo collisions with neutral atoms, PIC-MCC, IEEE Trans. Plasma Sci. 19 (1991) 65.

[16] E. Hairer, C. Lubich, G. Wanner, Geometric numerical integration illustrated by the Störmer/Verlet method, Acta Numerica (2003) 1-51.

[17] E. Hairer, C. Lubich, G. Wanner, Geometric Numerical Integration. StructurePreserving Algorithms for Ordinary Differential Equations, Springer, Berlin, 2002.

[18] B. García-Archilla, J. M. Sanz-Serna, R. Skeel, Long-time-step methods for oscillatory differential equations, SIAM J. Sci. Comput. 20 (1999) 930-963.

[19] M. Hochbruck, C. Lubich, A Gautschi-type method for oscillatory second-order differential equations., Numer. Math. 83 (1999) 403-426.

[20] W. Gautschi, Numerical integration of ordinary differential equations based on trigonometric polynomials, Numer. Math. 3 (1961) 381-397.

[21] A. Pukhov, Three-dimensional electromagnetic relativistic particle-in-cell code VLPL (virtual laser plasma lab), J. Plasma Phys. 61 (1999) 425. 